The University of Southern Mississippi

The Aquila Digital Community

Faculty Publications

10-1-1999

\title{
Pretransitional Behavior Above the Nematic-Isotropic Phase Transition of an Auxetic Trimer Liquid Crystal
}

\author{
Daeseung Kang \\ Case Western Reserve University \\ Milind P. Mahajan \\ Case Western Reserve University \\ Shiyong Zhang \\ Case Western Reserve University \\ Rolfe G. Petschek \\ Case Western Reserve University \\ Charles Rosenblatt \\ Case Western Reserve University
}

See next page for additional authors

Follow this and additional works at: https://aquila.usm.edu/fac_pubs

Part of the Chemistry Commons

\section{Recommended Citation}

Kang, D., Mahajan, M. P., Zhang, S., Petschek, R. G., Rosenblatt, C., He, C., Lu, P., Griffin, A. (1999). Pretransitional Behavior Above the Nematic-Isotropic Phase Transition of an Auxetic Trimer Liquid Crystal. Physical Review E, 60(4), 4980-4982.

Available at: https://aquila.usm.edu/fac_pubs/9262

This Article is brought to you for free and open access by The Aquila Digital Community. It has been accepted for inclusion in Faculty Publications by an authorized administrator of The Aquila Digital Community. For more information, please contact Joshua.Cromwell@usm.edu. 
Authors

Daeseung Kang, Milind P. Mahajan, Shiyong Zhang, Rolfe G. Petschek, Charles Rosenblatt, Chaobin He, Puwei Lu, and A.C. Griffin 


\title{
Pretransitional behavior above the nematic-isotropic phase transition of an auxetic trimer liquid crystal
}

\author{
Daeseung Kang, ${ }^{1}$ Milind P. Mahajan, ${ }^{1}$ Shiyong Zhang, ${ }^{1}$ Rolfe G. Petschek, ${ }^{1}$ Charles Rosenblatt, ${ }^{1}$ Chaobin He, ${ }^{2}$ \\ Puwei Liu, ${ }^{2}$ and A. C. Griffin ${ }^{2,3}$ \\ ${ }^{1}$ Department of Physics, Case Western Reserve University, Cleveland, Ohio 44106-7079 \\ ${ }^{2}$ Department of Chemistry \& Biochemistry, University of Southern Mississippi, Hattiesburg, Mississippi 39406 \\ ${ }^{3}$ Department of Polymer Science, University of Southern Mississippi, Hattiesburg, Mississippi 39406 \\ (Received 12 February 1999)
}

\begin{abstract}
Static light scattering and electric field-induced Kerr measurements were performed above the nematicisotropic phase transition of a terminal-lateral-lateral-terminal negative Poisson ratio trimer. For both measurements the inverse susceptibility was observed to be nearly linear with temperature, a result inconsistent with our previously reported Kerr data [Phys. Rev. E 58, 2041 (1998)]. [S1063-651X(99)11010-9]
\end{abstract}

PACS number(s): 61.30.Gd, 64.70.Md

Although most polymers exhibit a positive Poisson ratio, one can imagine cases where the Poisson ratio is negative, i.e., the polymer expands laterally when stretched [1,2]. This so-called "auxetic" behavior has been found in several polymers [2-6] and metal foams [5], and may be based on atomic scale mechanisms $[7,8]$ or an inverted honeycomb (reentrant cell) molecular structure $[1,3,9]$. He et al. have synthesized a new class of auxetic materials based upon siteconnectivity driven rod reorientation in main chain liquid crystalline polymers [10], which is schematically illustrated in Fig. 1 for the model trimer. For this class of molecules the terminal mesogens are connected at their ends, and the central mesogen at its core. In the nematic phase the molecules interact via a combination of anisotropic van der Waals and steric interactions, giving rise to a molecular arrangement in which the mesogenic axes are approximately parallel to each other [Fig. 1(a)]. For conformations in which the ends of the molecule are far apart, i.e., the spacer groups are fully extended, the central mesogen orients at a large angle, or even perpendicular, to the main chain direction [Fig. 1(b)]. X-ray results on a polymer that is based on this model trimer indeed show increased lateral separation between chains when the polymer fiber is stretched [10].

In a previous paper we examined the trimer shown in Fig. 2 [11]. In the nematic phase we measured the splay, twist, and bend elastic constants, finding values similar to that of typical monomers [12]. However, the ratio $K_{33} / K_{11}$ was slightly enhanced and the ratio $K_{11} / K_{22}$ was slightly reduced from that of typical monomers, especially near the nematicisotropic (NI) phase transition temperature $T_{\mathrm{NI}}$. We also examined the temperature dependence of the susceptibility $\chi$ in the isotropic phase via an electric-field-induced birefringence (Kerr) experiment. For most liquid crystalline materials the inverse susceptibility $\chi^{-1}$ is linear in temperature [12]. For the auxetic trimer, however, $\chi^{-1}$ was found to rise rapidly just above the transition, with its slope decreasing with increasing temperature. This behavior is shown in the inset of Fig. 3. The results were explained in terms of a Landau model based on separate but coupled order parameters, one order parameter for the two terminal mesogens and another order parameter for the core mesogen. In this paper we re- visit the pretransitional behavior above the nematic-isotropic phase transition using two mutually independent probes, viz., light scattering and Kerr measurements. Our central result is that both measurements exhibit mean-field-like behavior, i.e., $\chi^{-1}$ is linear in temperature. These results are not consistent with our previously reported Kerr measurements, results that we believe were compromised by faulty temperature control.

The trimeric liquid crystal was prepared by a multistep synthetic route [13]. The compound was subjected to the standard chromatographic and spectroscopic techniques for purity and structure determination. Unlike the polymer, the trimer flows easily, with a relatively low viscosity that is not much different from that of typical low molecular weight nematics [12].

We first reexamined the susceptibility using the electricfield-induced birefringence (Kerr) technique. Because of the relatively high phase transition temperature $T_{\mathrm{NI}}$, we suspect that there may have been a systematic artifact that affected our previously reported measurements [11]. In those measurements the temperature went well beyond the desirable operating region of the temperature controller and sensing thermistor. For these experiments we modified the thermal apparatus to handle temperatures between $150{ }^{\circ} \mathrm{C}$ and $200{ }^{\circ} \mathrm{C}$, and repeated our earlier measurements. A pair of clean, untreated, indium-tin-oxide-coated glass slides was separated by a $6-\mu \mathrm{m}$ Mylar spacer and cemented together with high temperature epoxy (rigid to $220^{\circ} \mathrm{C}$ ). The thickness of the cell was measured by an interference method [14] and determined to be $6.5 \pm 0.2 \mu \mathrm{m}$. The cell was placed into an

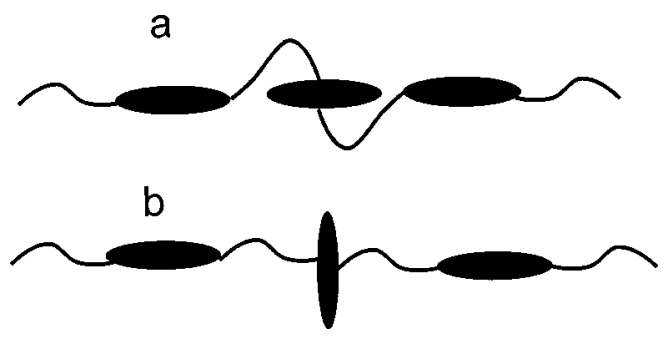

FIG. 1. Schematic representation of auxetic trimer. (a) Nematic phase. (b) Stretched molecule with extended spacer groups. 


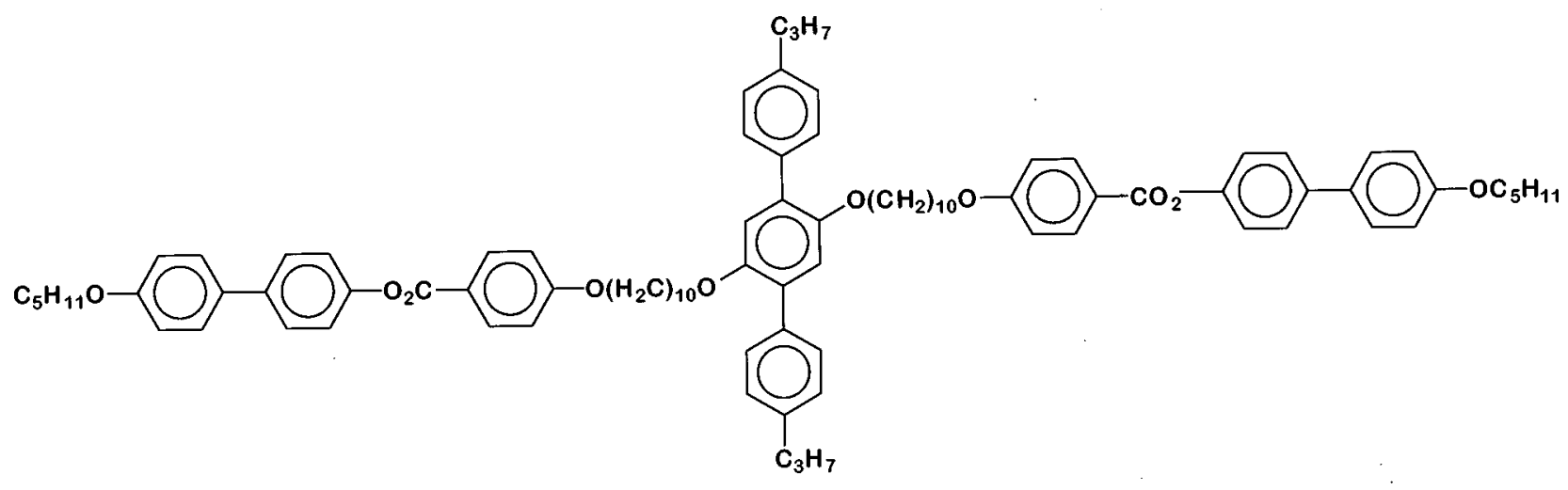

FIG. 2. The structure of the auxetic trimer.

improved temperature controlled oven, stable to $10 \mathrm{mK}$. The optical arrangement and method of data analysis are described in detail in Ref. [11]. The liquid crystal was driven with an a.c. voltage at frequency $f=1000 \mathrm{~Hz}$ and the resulting $2 f$ component of birefringence $\Delta n$ was measured as a function of electric field $E$. Figure 3 shows $\left(d \Delta n / d E^{2}\right)^{-1}$ vs temperature. Unlike the previously reported data (shown in the inset of Fig. 3), the new data are nearly linear in $E^{2}$. The small deviations of $\chi^{-1}$ from linearity at high temperature are most likely due to conformational changes of the molecule, as well as experimental noise when measuring small values of $\chi$ in the region well above $T_{\mathrm{NI}}$. Finally, note that the temperatures of the phase transition for the new data and old data (inset) are offset by approximately $5^{\circ} \mathrm{C}$, a consequence of our faulty temperature control in the earlier experiment.

Given the nearly linear behavior of $\chi^{-1}$ vs temperature, we next examined orientational fluctuations in the isotropic phase by means of light scattering. Although the fluctuations may be characterized by measuring either the intensity of the

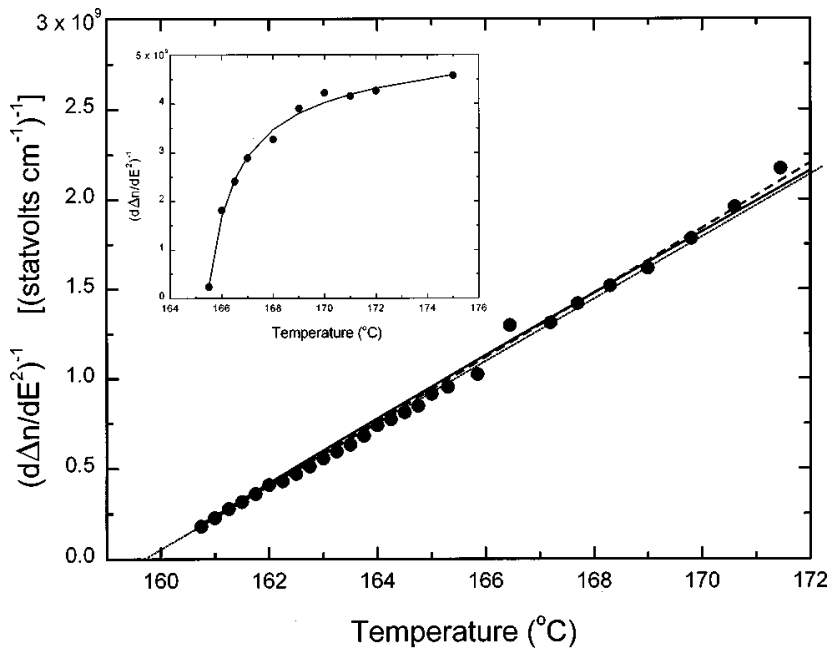

FIG. 3. Inverse Kerr coefficient $\left(d \Delta n / d E^{2}\right)^{-1}$ vs temperature. The dotted line represents the linear fit, and the dashed and solid lines represents behavior predicted by a two-order-parameter model with different sets of parameters (see text). The inset corresponds to data from Ref. [11]. Note that the two temperature scales differ because of a faulty temperature control in Ref. [11]. scattered light or the relaxation time $\tau$ of the intensity autocorrelation function, we opted for the static measurement because $\tau$ is of order $1 \mu \mathrm{s}$, which is near the limit of our instrument. A glass capillary tube of inner diameter $1.5 \mathrm{~mm}$ was filled with the liquid crystal and its end was sealed by flame heating. The tube was then inserted into a rectangular cuvette filled with Dow Corning 550 silicon oil. The oil served as an index matching fluid to avoid scattering that would otherwise occur from a capillary-air interface. Both the liquid crystal and the silicon oil were filtered to remove dust using a $0.22-\mu \mathrm{m}$ Millipore filter. The cuvette was transferred to an oven that was temperature controlled to $10 \mathrm{mK}$, and the scattering apparatus is described in Ref. [15]. The depolarized intensity at a scattering angle of $90^{\circ}$ was measured by inputting the signal from the photomultiplier into a pulse amplifier-discriminator, whose output was fed into a digital autocorrelator. The temperature of the oven was first stabilized at $175^{\circ} \mathrm{C}$, and electronically ramped downward at the rate of $0.2^{\circ} \mathrm{C} \mathrm{min}^{-1}$. The intensity of the scattered light [proportional to the count rate] was recorded by the correlator in the raw intensity mode, and the inverse of the intensity vs temperature is plotted in Fig. 4.

According to the Landau theory [16], the mean square order parameter fluctuation at a given wave vector $\vec{q}$ is given

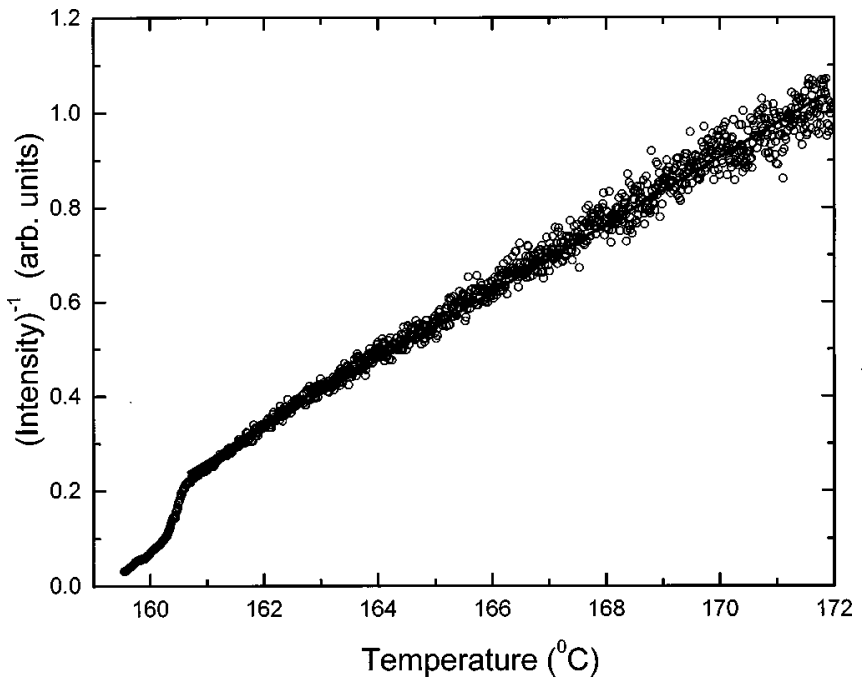

FIG. 4. Inverse intensity vs temperature from light scattering measurement. The solid line is meant as a guide for the eye. 
by $\left\langle\overleftrightarrow{Q}^{2}(\vec{q})\right\rangle=(k T / V A)\left(1+\xi^{2} q^{2}\right)$, where $\overleftrightarrow{Q}$ is the tensor order parameter of the nematic phase, $A=a\left(T-T^{*}\right)$ is the coefficient of the term quadratic in $\overleftrightarrow{Q}$ of the free energy expansion, $T^{*}$ is the supercooling temperature of the isotropic phase, $V$ is the volume, and $\xi$ is the correlation length for nematic fluctuations. For visible light and $90^{\circ}$ scattering, the quantity $\xi^{2} q^{2} \ll 1$, and thus the inverse of intensity is expected to be approximately proportional to $\left(T-T^{*}\right) / T$. The data in Fig. 4 are clearly consistent with this mean-field behavior and with the new Kerr data in Fig. 3. A line is drawn through the data to guide the eye and to emphasize its traditional mean-field-like character. Three caveats are in order. First, at higher temperatures there is a small downward curvature to the data that comes about because of unavoidable minute quantities of dust in the scattering cell. Second, in the region just above $T_{\mathrm{NI}}$ there is also a small amount of curvature; this behavior is due to correlation effects and is well known [17]. Finally, we note that the rounding that occurs over $200 \mathrm{mK}$ near the phase transition is likely due to a combination of a small, impurity-driven biphasic region and, more importantly, to steady-state temperature gradients in the cell that occur at these very high temperatures.

In our earlier paper we speculated that the highly nonlinear behavior of $\left(d \Delta n / d E^{2}\right)^{-1}$ vs temperature could be described by a Landau model involving (for simplicity) scalar order parameters that are coupled [11]. We supposed that $S_{1}$ corresponds to the order parameter of the two end mesogens and $S_{2}$ to the order parameter of the central mesogen, and wrote the most general phenomenological Landau free energy for a scalar order parameters as

$$
F=a S_{1}^{2}+b S_{1} S_{2}+c S_{2}^{2}-e_{1} S_{1}-e_{2} S_{2},
$$

where $a, b$, and $c$ may each have a unique temperature dependence and $e_{1}$ and $e_{2}$ are electric field factors. After some algebra, we obtained the inverse Kerr coefficient in the form

$$
\left(\frac{d \Delta n}{d E^{2}}\right)^{-1}=\left[\frac{A}{T-T_{11}}+\frac{B}{T-T_{12}}\right]^{-1},
$$

where $A, B, T_{11}$, and $T_{12}$ are constants. This form successfully fitted our earlier data (cf. Fig. 3 inset). Because the environments felt by the core and terminal mesogens are quite different, we believe that it is still reasonable to fit the present data with this form. In addition to the traditional linear fit (dotted line) in Fig. 3, we show two possible fits to Eq. (1): The solid line represents the inverse Kerr coefficient using the two-order-parameter model with parameters $A$ $=5.4 \times 10^{-9} \mathrm{cgs}, \quad B=1.0 \times 10^{-9} \mathrm{cgs}, \quad T_{11}=159.7^{\circ} \mathrm{C}$, and $T_{12}=130.0^{\circ} \mathrm{C}$; and the dashed line using $A=5.7$ $\times 10^{-9} \mathrm{cgs}, \quad B=-0.4 \times 10^{-9} \mathrm{cgs}, T_{11}=159.7^{\circ} \mathrm{C}$, and $T_{12}$ $=130.0^{\circ} \mathrm{C}$. It is important to recognize that with a fourparameter fit, the $\chi$-square surface has many shallow minima, and the parameters are extremely sensitive to small variations in the data. We thus conclude that despite the apparent "traditional" single-order parameter mean-field-like Kerr results, the terminal mesogens and core mesogen may actually be best modeled by two distinct (but coupled) order parameters. In fact, one of the order parameters-this would be $S_{2}$ of course-may even be slightly negative (cf. dashed line), consistent with the molecular architecture. It is clear, however, that optical experiments are not completely suitable to elucidate this conjecture, and the multiple order parameter approach would be better investigated by, e.g., a nuclear magnetic resonance probe. This work is planned in the near future.

To summarize, we have experimentally observed that the pretransitional behavior above $T_{\mathrm{NI}}$ of the auxetic trimer shown in Fig. 2 is apparently similar to that of typical monomeric liquid crystals. We have also shown, however, that within experimental error the Kerr data may be described by the two-order-parameter model introduced in another publication [11]. As the spacer groups may be made shorter or longer, it is conceivable that qualitatively different behavior may be observed, especially for shorter (and therefore less flexible) spacer groups.

This work was supported by the National Science Foundation under Grant Nos. DMR-9502825 and DMR-9420843, by NSF's Advanced Liquid Crystalline Optical Materials Science and Technology Center under Grant No. DMR8920147, and by the U.S. Air Force Office of Scientific Research under Contract No. F49620-94-1-0454.
[1] K. E. Evans, I. J. Hutchinson, and S. C. Rogers, Nature (London) 353, 124 (1991).

[2] R. S. Lakes, Science 235, 1038 (1988).

[3] L. J. Gibson and M. F. Ashby, Cellular Solids: Structure and Properties (Pergamon, Oxford, 1988), pp. 70-82.

[4] K. E. Evans, Endeavour 15, 170 (1991).

[5] E. A. Friis, R. S. Lakes, and J. B. Park, J. Mater. Sci. 23, 4406 (1988).

[6] K. L. Alderson and K. E. Evans, Polymer 33, 4435 (1992).

[7] A. E. H. Love, A Treaty on the Mathematical Theory of Elasticity, 4th ed. (Dover, New York, 1944).

[8] D. J. Gunton and G. A. Saunders, J. Mater. Sci. 7, 1061 (1972).

[9] G. Wei and S. F. Edwards, Comput. Polym. Sci. 2, 44 (1992).
[10] C. He, P. Liu, and A. C. Griffin, Macromolecules 31, 3145 (1998).

[11] D. Kang, M. P. Mahajan, R. G. Petschek, C. Rosenblatt, C. He, P. Liu, and A. C. Griffin, Phys. Rev. E 58, 2041 (1998).

[12] See, e.g., S. Chandrasekhar, Liquid Crystals (Cambridge University Press, Cambridge 1992).

[13] P. Liu, C. He, C. J. Booth, and A. C. Griffin, Abstr. Pop. Am. Chem. Soc. 216, U108 (Part 3) (1998).

[14] C. Rosenblatt, J. Phys. (Paris) 45, 1087 (1984).

[15] G. A. DiLisi, C. Rosenblatt, A. C. Griffin, and U. Hari, Phys. Rev. A 45, 5738 (1992).

[16] L. D. Landau, and E. M. Lifshitz, Statistical Physics (AddisonWesley, Reading, MA, 1958).

[17] T. W. Stinson, J. D. Litster, and N. A. Clark, J. Phys. Colloq. 33, C1-69 (1972). 